\title{
Engaging MSM Communities in HIV Self-Testing Research and Programming in Kenya: Collaborations, Tacit Knowledge, and a Community- Based Programme Science Approach
}

\section{Bernadette Kina Kombo ( $\nabla$ kombob@myumanitoba.ca )}

University of Manitoba Faculty of Health Sciences https://orcid.org/0000-0003-1808-4356

\section{Matthew Thomann}

University of Maryland at College Park

\section{Lisa Lazarus}

University of Manitoba Faculty of Health Sciences

\section{Helgar Musyoki}

NASCOP

\section{Kennedy Olango}

Men Against AIDS Youth Group (MAAYGO)

\section{Samuel Kuria}

Mambo Leo Peer Empowerment Group (MPEG)

\section{Martin Kyana}

HIV \&AIDS People's Alliance of Kenya (HAPA Kenya)

\section{Memory Otieno}

Partners for Health and Development in Africa

\section{Margaret Njiriani}

Partners for Health and Development in Africa

\section{Janet Musimbi}

Partners for Health and Development in Africa

\section{Parinita Bhattacharjee}

University of Manitoba Faculty of Health Sciences

\section{Robert Lorway}

University of Manitoba Faculty of Health Sciences

\section{Research}

Keywords: Community-based programme science, men who have sex with men, Kenya, HIV self-testing, community involvement 
Posted Date: October 26th, 2021

DOI: https://doi.org/10.21203/rs.3.rs-861094/v2

License: (c) (i) This work is licensed under a Creative Commons Attribution 4.0 International License. Read Full License 


\section{Abstract}

Background: Understanding the basic principles for achieving research outcomes that are relevant to local communities is invaluable. The process allows for building of collaborative spaces and redistribution of power in knowledge production practices. This is because members of marginalized and vulnerable communities have long been critical of a research culture that has historically failed to advance meaningful collaboration and overlooks local forms of knowledge at various phases of the research processes. There has however not been a clear framework that outlines how communities should be meaningfully engaged. Rather, substantial variations in the nature of community engagements exist across research projects, thus requiring a more nuanced approach of working with communities.

Methods: In this methodological paper, we describe how a community-based program science approach guided an interdisciplinary research project to inform the rollout of HIV self-testing (HIVST) among men who have sex with men (MSM) in three urban counties in Kenya. Community members and their research and programmatic partners collaborated through all phases of the research process including research design, data collection, and translation of research. Importantly, community researchers and policy makers also played an integral role in data analysis, going well beyond the conventional role of "community engagement" in global health research.

Results: The study created a platform that enabled meaningful collaborations across the diverse stakeholders and allowed the MSM communities to contribute to the decisions and solutions impacting on their community. Our community-based program science approach recognizes that community researchers possess tacit knowledge, a form of expertise that formally trained researchers do not have.

Conclusion: We argue that in order to fully engage with such expertise, marginalized communities must be meaningfully involved in all levels of evidence building and decision making around programs impacting their communities. We equally emphasize the importance of setting up operating norms and clear definitions of partnership roles at the initial stages of establishing collaborations. Such a move gives the community a chance to trust their capabilities and contribute more meaningfully throughout the research processes.

\section{Background}

Members of marginalized and vulnerable communities have long been critical of a research culture that has historically failed to advance meaningful collaboration and overlooks local forms of knowledge at various phases of the research processes. Communities and researchers alike have called for the adoption of decolonizing research processes which ideally should address the issues of power imbalances and mistrust in research, while allowing for contextualized, respectful and legitimate research practices that build on community agency $(1-4)$. For more than two decades, researchers have adopted community-based research (CBR) methods that strive toward the equitable involvement of community members, organisations, researchers and other key partners in all aspects of the research process leading 
to shared decision making and ownership of data between community members and academic investigators (5-9). Although the underlying purpose and specific characteristics of CBR are somewhat contested (10), CBR generally calls for community-driven projects that foster active collaboration and participation at every stage of research, co-learning between scientists and community partners, the dissemination of research findings, and tailoring of interventions that are culturally appropriate (11). CBR may contribute to research trustworthiness as it has been shown to not only strengthen the research process but also enhance research integrity and growth of collaborations in science as a whole, thereby increasing its relevance to the community (12-14).

Notwithstanding the strengths of CBR, communities and researchers have at times challenged the overall implementation of the approach. For instance, the lack of a clear framework for implementing CBR leaves the interpretation of its principles at the discretion of the researchers, leading to different interpretations of the role of the community in research and substantial variation in the nature of community involvement across projects $(15,16)$. Often, the level of community involvement stagnates significantly after seeking input from community advisory groups and in some instances training the community to collect data within their communities, but largely fails to engage community participation across the life course of the research process. Moreover, in as much as CBR is promoted as an approach that can facilitate translation and dissemination of research findings to the community, in practice, there are notable gaps in the distribution of responsibilities, particularly during the dissemination of study findings. Often, researchers focus on scientific publications while community stakeholders use the data primarily for lobbying and advocacy (15). This then impacts the translation of research findings into practice as the community is likely to perceive research publications as a purely academic exercise and not incorporate the findings into programs. To address these shortcomings, Brizay and colleagues have recommended a more nuanced way of working with communities, guided by a framework outlining the role of each stakeholder in the different steps of the research process ${ }^{11}$. The framework should be one that will provide for flexibility in the definition of the different partners' responsibilities based on their capacities but at the same time providing a structure to work in. They see this as a way of achieving a greater role for community in the planning and implementation of interventions and to ensure a collaborative process of generation and uptake of research findings within routine practice (15).

Program science, a research paradigm that involves an iterative process of putting evidence generated from research into practice as well as generating evidence from the routine operation of programs, (1720) can further build on the collaborative approach proposed by Brizay and colleagues (15). It involves embedding research processes into the operation of programs, so that scientific knowledge continually feeds out from and into the program context to inform strategic planning, program implementation and program management (18). Put simply, program science is the science of programs as well as the science for programs. Within program science, collaborative engagements between state health systems, local and international NGOs, funders and academic institutions are conceptualized as working in alignment toward the goal of improving the health of populations. 
The integration of a CBR and program science approach has the potential to foster stronger research collaborations with communities in the production and utilization of evidence throughout the life of programs. The emerging field of community-based program science (CBPS) refers to the unique linking of these two complimentary research paradigms (19). CBPS directly involves communities in processes of knowledge production and sustained negotiations with health and social service institutions over the life course of interventions. Importantly, it recognizes the roles played by power and social inequalities in the unequal production of expert knowledge, and centers the importance of lay expertise and tacit knowledge (21). Using a case study approach, we describe a pragmatic operationalization of CBPS by drawing on a Bill \& Melinda Gates Foundation-sponsored collaborative HIV self-testing study (HIVST) among men who have sex with men (MSM) in three diverse counties in Kenya.

\section{The MSM HIVST Study}

MSM shoulder a disproportionate burden of HIV in Kenya, with an HIV prevalence 4-5 times higher than that of the general population (22-24). In 2018, the National AIDS and STI Control Programme (NASCOP) led a key populations mapping and size estimate exercise in selected counties that showed that there were approximately 33,000 MSM frequenting hotspots in Kenya (25). According to the Kenya HIV Prevention Response and Modes of Transmission Analysis performed by the National AIDS Control Council (NACC) in 2008, $15.2 \%$ of new HIV infections in Kenya were attributed to MSM and people in prisons(26). In a later study through the integrated biological and behavioural surveillance survey, MSM had a HIV seroprevalence of $18 \%$.(27) However, most MSM in Kenya remain unaware of their HIV status, due to high levels of stigma, limited or a lack of MSM-friendly health services, confidentiality concerns, and a fear of blood/finger prick testing 28,29.

As is the case in 53 other countries on the African continent, homosexuality is criminalized by the Kenyan penal code, a relic of colonial-era laws that were upheld by the Kenyan high court in 201930-34. While data from Kenya is limited, delayed HIV testing due to anticipated stigma is well-documented among MSM and other sexual and gender minorities in countries with a range of legal stances on homosexuality 35-39. Because of estimated high rates of MSM who are unaware of their HIV status, research has called for innovative approaches to reach individuals who are undiagnosed40,41. HIV self-testing (HIVST) - a process in which an individual performs and interprets their own HIV test in a setting of their choosing has been viewed as a promising strategy for reducing the number of undiagnosed cases of HIV among vulnerable populations such as MSM, particularly in contexts where stigmatizing social conditions are likely to result in low levels of engagement with facilities-based HIV testing services 42-46. Moreover, evidence shows that HIVST has the potential to improve early detection of HIV and can facilitate earlier linkage with HIV prevention and treatment services 47,48. In Kenya, a number of studies have been conducted on the feasibility of HIVST scale-up among serodiscordant couples and female sex workers (FSW) 49-54. However, evidence is extremely limited 55, on how, when, and in what contexts HIVST delivery to MSM can increase knowledge of HIV status and lead to early linkage to treatment and prevention services. 
Integrating HIVST interventions with CBPS principles in which MSM are at the center of program planning and implementation ensures a platform that empowers them to contribute to decisions and solutions that impact their communities. We undertook a mixed methods CBPS research project to design and implement HIVST programs among MSM in three diverse counties in Kenya (Kisumu, Mombasa, and Kiambu). The study was initiated through a collaboration between the University of Manitoba (UM), Partners for Health and Development in Africa (PHDA), the National AIDS and STI Control Programme (NASCOP), G10 (an MSM research network in Kenya), and MSM-led community-based organizations (CBOs) in each of the three counties (Mamboleo Peer Empowerment Group (MPEG) in Kiambu, Men Against AIDS Youth Group (MAAYGO) in Kisumu, and the HIV \& AIDS People's Alliance of Kenya (HAPA Kenya) in Mombasa). The study was embedded within existing HIV and sexual health programs implemented by the selected CBOs, who run HIV prevention and treatment programs that specifically cater to MSM and therefore offered a crucial partnership for the delivery of HIVST. The details of the larger intervention study have been previously described56. In this paper we describe the CBPS process, focusing on the specific qualitative components of the study.

\section{Methods}

\section{Developing a CBPS Approach for the MSM HIVST Study Setting the research agenda within existing programs}

In 2018, after examining program data that revealed low HIV testing rates among MSM, NASCOP and University of Manitoba initiated discussions between partners on the potential of initiating a CBPS HIVST study among MSM. Kenya has a history of strong leadership among organizations serving MSM, including a national body (G10) that leads research among LGBTI communities. NASCOP, in partnership with UM and PHDA, approached G10 and its partners in Kiambu, Kisumu and Mombasa of which G10 identified three partner $\mathrm{CBO}$ s to participate in the project. The $\mathrm{CBO}$ s each had extensive experience in program implementation and a large program reach. We developed a formal partnership with each $\mathrm{CBO}$, initially through the exploratory research phase and then through the entire research process from protocol development/research design, data collection, data analysis, and dissemination/translation of findings. We began by setting up a three-month exploratory process to assess the feasibility of the study. Based on the successful completion of the preliminary assessment phase which entailed virtual mapping58 (assessing MSM use of virtual platforms such as geosocial networking and other social networking applications to find sexual partners) and consultations with the community to assess feasibility of partnerships, we negotiated formal, 12-month partnership agreements with clear roles and defined responsibilities for all collaborators at all the levels of the study. After consensus and consultation, UM was given the responsibility of overseeing the evaluation, the CBOs took the role of study implementation, while NASCOP was given the role of policy and advocacy. Point persons from each institution were selected and formed a core team of 16 members for oversight and decision-making. Though leadership roles were clearly divided, supportive roles were also defined. For instance, while UM 
served as the lead on the evaluation, the CBOs participated in the design of the study, provided direct guidance and support to UM by identifying community researchers (CRs), supported and monitored data collection, supported analysis and interpretation of the data, and used the data for improvement of programs. Similarly, though CBOs led the implementation, UM and NASCOP contributed to the study design by co-designing the intervention design, conducting capacity building on HIVST and modes of distribution, training of CRs in data collection and analysis, and designing a monitoring system. In addition, UM and its local partner PHDA had a representative based in each of the participating counties who then became the point person for project-related discussions and decision-making. Regular updates occurred through monthly report sharing, face-to-face meetings, email updates and more informal approaches. Importantly, UM, PHDA and the CBOs supported NASCOP in the dissemination by sharing study findings in key forums such as the Kenya's key populations community of experts forum, and advocating with the different county governments to share study findings and organize collaborative activities.

\section{Study Design and Protocol Development}

Collaborative strategic planning in central to CBPS. It centers on ensuring all key stakeholders in a study are actively involved including in the design and the development of a project. The process allows for the integration of tacit knowledge and the adaptation of local contexts in the design of projects. In our case, to ensure active participation of the MSM community and all other collaborating partners in the entire research process, a protocol development workshop was organized. The workshop saw all the collaborating partners come together in order to establish the research questions, the rationale of the study project, and strategies that would be relevant in the conduct of the study. As such the aim of the workshop was for each of the collaborating partners including the MSM community to develop a study protocol to evaluate a community-based HIV self-testing delivery strategies on reducing undiagnosed HIV infection, and improving linkage to prevention and treatment services, among men who have sex with men in Kenya.

\section{Baseline Qualitative Exploratory Study}

\section{Recruitment and Training of Community Researchers}

As part of the ongoing active involvement of the community in the study, the $\mathrm{CBO}$ s, in collaboration with the PHDA field coordinators and the qualitative research coordinating team (which included the first, second and the last author), recruited CRs from within their membership who would then be involved in the qualitative data collection and analysis processes throughout the study period. Four CRs from each of the three participating sites were recruited, for a total of twelve CRs. For the baseline qualitative exploratory phase, the CRs, together with their respective community study supervisors, were taken through a participatory training on key principles in research ethics 59 and qualitative research methodology by the first, second, and last author. In the same forum, the CRs participated in the framing of the interview questions and development of the data collection tools, including the interview guide and demographic form. In order to have precise questions that could be asked in different local languages 
across the three participating sites and to troubleshoot for potential points of confusion for participants, the CRs practiced the questions through multiple role-play sessions and refined them accordingly. For example, during the first role play session a CR from Mombasa asked about the impact of bleeding gums on the test. The second author captured this exchange in a fieldnote included below.

While the community researchers practiced using the interview guide with one another, Fatima raised her hand and asked, "What happens if their gums are bleeding and there is blood on the stick?" The coordinating research team initially thought that this would not present a problem as the test is designed to identify antibodies in saliva. However, as we carefully re-read the clinical information on the back of the instructions, we discovered that blood on the test strip can indeed lead to an invalid test. This also raised the question among some community researchers about how to explain to participants that though the test works by identifying antibodies in saliva, HIV cannot be transferred through saliva. [The last author] stopped the role-playing exercise to stress that this would be an instance in which the community researcher has an ethical responsibility to ensure that the participant does not leave with incorrect information. After some discussion, the interview guide was edited to ensure that when the community researcher explains the oral swab, they also explicitly state that HIV is not transmitted through saliva and to remind the participant that they can ask more detailed information from the clinician after the interview process.

\section{Sampling}

Before the CRs began conducting interviews in their respective counties, the first, second and last author together with the PHDA field coordinators visited each of the respective sites where they advised CRs and observed them as they created their own sampling tree (see Fig. 1). Using pseudonyms, they listed their potential participants while considering both those enrolled and not enrolled in programs, as well as ensuring representation from different socio-economic backgrounds. Inclusion criteria included men reporting having had sex with another man or transgender woman in the past 12 months, being 18 years or older, and willing to be followed over a period of one year to participate in baseline, midline and endline interviews. Each CR team generated a list of potential participants who met the inclusion criteria from within their own social networks. Peer educators or outreach workers in the CBOs were not eligible to participate in the interviews. Participants were selected to ensure equal age distribution, as well as to account for the experiences of MSM who were enrolled in CBO programs and those who were not. To ensure representation of different socio-sexual networks, CRs also selected participants based on their involvement in sex work and their socio-economic status. Part of this process was captured by the second author in the fieldnote below:

When introducing the concept of sampling to the Mombasa team, [the last author] used the metaphor of cooking rice. "In order to know if it is cooked well you need to taste different spots in the pot. Sampling in research is the same idea," he explained. We asked the community researchers to start by listing the "different kinds of MSM" in Mombasa. They listed: age, sex work, enrolled [in MSM programming] vs. not enrolled, and "class". The last author mentioned that this is essentially a conversation about diversity; that while they are all MSM, there are characteristics that make them different. 


\section{Conducting the interviews}

Between May and August of 2019, the CRs interviewed a total of 72 participants. The interviews explored participants' perceptions on the HIVST technology, and their opinions on the best strategies CBOs could use to deliver HIVST to the MSM community. With consent from all participants, the interviews were audio recorded, and later translated [when necessary] and transcribed verbatim in English by trained community members. The study procedures were approved by the research and ethics review board at the Kenyatta National Hospital in Kenya and the University of Manitoba, and all participants provided written informed consent.

\section{Participatory Data Analysis}

One key principle of CBPS-driven interventions is having communities actively participate in the production of evidence throughout the life course of the intervention. However, more often than not, studies that profess to follow a CBR approach fail to directly include community participation in the data analysis process 60-62. Engaging community members in the analysis and interpretation of research data ensures communities' perspectives, knowledge, and contributions are authentically included. The process yields rich analytic insight and contextualization that fosters the translation of findings into local community health practice. Local knowledge gained through years of experience and practice as a member of the community - what is referred to as tacit knowledge - is an integral dimension in the interpretation and translation of findings into practice within the local context 63 . We therefore undertook a deliberate move to involve the $\mathrm{CRs}$ and their $\mathrm{CBO}$ s study coordinators in a collaborative analysis process of the qualitative interview data. The team participated in a process of meaning-making that had multiple steps64,65.

\section{Training on Thematic Analysis}

Under the guidance of a locally-based qualitative research coordinator (the first author) and two senior medical anthropologists (the second and final authors), CRs were taken through a two-day training to learn how to conduct basic thematic analysis from an interpretivist perspective 66. Specifically, using different facilitation skills and simplified visual aids such as videos (https://www.youtube.com/watch? $\mathrm{v}=\mathrm{phXssQBCDIs}$ ) and samples of already analyzed transcripts, the team learned about reviewing and familiarization with the transcripts, coding, memo-ing, data interpretation, and composing findings section. Three transcripts from each of the three participating sites were picked for the participatory data analysis exercise.

\section{Familiarization and Coding}

To embark on the analysis process, the CR team undertook a deep reading of a set of transcripts in small groups in order to familiarize themselves with the contents of the transcripts. They then provided an overview of the data to the larger research team before returning to their small groups to begin a more detailed analysis of the individual sections of the transcripts. At this stage, the CRs read through the 
transcripts a second time and took initial notes. For this first step, team members were put in smaller groups composed of both the CRs and study coordinators. The first, second, and final authors supported the groups by listening to the various discussions and asking questions that would be relevant to aid the analysis process. The purposeful grouping in turn allowed for a variety of perspectives to be represented during the research team's early interaction with the transcripts and ensured a cross-mingling of ideas. For example, one CR from Kisumu directed his small group's attention to the complex security concerns that the kit posed. A fieldnote captured by the second author details this exchange:

While reviewing a section of the transcript, John turned to [second author] and [fourth author] and said that the interviewer "missed" something. While being probed for their concerns about the HIVST, the participant had briefly alluded to security concerns, expressing the potential for the kit "outing" users as they have become increasingly associated with the [MSM] community. John felt that the CR conducting the interview should have asked for more details about this and explained that several of participants he had interviewed had expressed similar security concerns. As the kits are marketed towards sexual and gender minorities, he worried that the mere presence of the kit might make users vulnerable to blackmail. [The fourth author] explained that the same thing happened when pre-exposure prophylaxis [PrEP] was marketed towards the community, making CBOs serving the community more visible than they had been previously. "Now they know where gay men are and where they access services," he explained.

During the coding process, the CR teams highlighted salient segments of the transcript and allocated a single word or short phrase to describe the content. The transcripts were printed with wide margins on the right side to create space to write codes on the transcripts.

\section{Code Grouping and Definitions}

During the next level of analysis, the small groups listed all the codes (child codes) and identifying related codes that they then grouped together to create parent codes. The parent codes were then presented to and discussed with the entire team. The discussion helped the team to begin to see some commonalities in the data and relationships across the different data sets. The members regrouped into three larger groups representing their three study sites. The CRs and the study coordinators then developed definitions for each parent code. Figure 2 shows a sample of listed codes from one of the groups.

\section{Reconciling Coding Schemes and Composing Findings Sections}

Next, the training facilitators reviewed the grouped codes and definitions in order to create a sample guide. The sample included the parent code, the definition of the code, and excerpts from the transcripts. Retaining the same site level groups, members used a laptop to extract excerpts from the three transcripts from their respective sites and assigned them accordingly to the defined parent codes. Each group then selected six parent codes they considered a priority and wrote up summaries bringing out the overall message of each of the parent codes that they would want to convey to program implementers and policy makers. From the combined codes, the team prioritized the following parent codes: 1) attitudes 
towards HIVST kits; 2) perceived advantages of using HIVST kits; 3) barriers that would impact on use of HIVST kits; 4) HIVST demand creation and advocacy; 5) service delivery approaches; and 6) role of health facilities. To conclude the analysis exercise, the groups exchanged their write-ups for critical review and the feedback provided to each site level group was used to improve the write-up of the findings.

\section{Dissemination Of Study Results}

A key feature of CBPS is the dissemination of study findings with community members and the active involvement of the community partners in the process. This is an approach therefore that we embraced in the implementation of our CBPS study. The MSM community were actively involved in the dissemination of study findings that went beyond the ordinary scientific publications as is often the case. We built on both existing and new structures in order to share the findings. For instance, the community was largely involved in the sharing of the findings at the counties and at national level through various forums such as the key populations committees of experts' forums and other relevant meetings. Also, the community wrote a first authored paper that shared with a wider audience on the efforts the community put to reach the MSM with HIV testing services amid Covid-1967.

\section{Results}

\section{Application of Findings in the HIVST Intervention}

The research team's findings on how to implement a community-led delivery of HIVST for MSM informed the intervention such that the CBOs included HIVST into their ongoing HIV prevention and treatment programs (for further details on the findings see Thomann et al., forthcoming). The data analysis process concluded with an agreement that individual site teams would come together at their respective organizational level, while considering their respective contexts, to review findings and to assess what could be integrated into the HIVST intervention for MSM. For instance, in the analysis, participants from across the sites had suggested MSM-specific social events, alongside a need for sensitization and networking at physical hotspots and virtual platforms, as a means to increasing uptake of HIVST kits. Emmanuel, a 37-year-old gay man from Kisumu, explained how MSM can be reached in groups and sensitized about HIVST.

...they can have theme activities that they partake like they have movie Mondays where people come to watch a movie, that is. .. a movie that you know has a gay-related theme or health-related or HIV healthrelated theme; so, they can bring their clients in those themed days, and they can be educated on that [HIVST].

The CBOs put in place mechanisms for holding MSM-themed social events that were designed to fit their specific contexts. For instance, in Kisumu, MAAYGO held what they referred to as an "HIVST Party" targeting their community members in a hotel and integrated themed activities such as singing, poetry, spoken word and run-way modelling. In Kiambu, MPEG held their MSM-themed event to promote HIVST 
at local entertainment clubs known to be frequented by MSM in the area. Similarly, mobilizations for the uptake of the kits occurred at physical spots frequented by MSM, as well as through varied social and dating virtual platforms such as Facebook pages, WhatsApp groups, Planet Romeo, Badoo and Grindr, among others.

The site-level analysis of the data by the different site teams provided instrumental findings that informed programs on the specific considerations they needed to make in the design of the intervention. The team from Mombasa, for instance, concluded from the analysis process that reaching some MSM in their region with HIVST kits would be deterred by the strong religious ties in the area and high levels of HIV-related stigma from religious leaders and the local community. During his interview, Bruce, a 21-yearold gay man from Mombasa, explained the deep-rooted HIV-related stigma within religious communities.

In Mombasa, because of churches, mosques, you cannot go just anywhere distribute [HIVST kits], they will look at you ... and at the streets when you just say we are here to teach you about HIVST kit, when you just name, the word HIV, people will be like looking at you, they will be surprised to hear the name, because HIV to some is like they believe that when I am infected with HIV that's the end of me.

In order to pro-actively address these stigma-related concerns, the Mombasa team engaged key religious leaders and other community opinion leaders from the area for a sensitization session on HIVST among MSM, ahead of distributing the HIVST kits. This provided a platform to initiate a partnership that would begin to address HIV-related stigma directed toward the MSM community.

Another area considered for HIVST interventions was the integration of MSM-targeted health services within government health facilities. Most participants interviewed who were not members of any of the MSM programs reported they would not want to be identified as an MSM or be associated with the MSMled organisations and preferred a distribution approach that would allow them to get the kits from government health facilities. Evans, a 35-year-old gay man from Kisumu, justified why he would prefer a government facility:

I have never had a problem taking services at the government hospital, yeah only if it's a sensitive issue like maybe anal STIs, that one I cannot go to the government hospital but when it's just the, the normal health routine like maybe I want to go for HIV testing and counselling or maybe I need to get some condoms, that one I don't have a problem, because usually when I go to access health in any health institution, I go as a Kenyan who has a right to access health. I don't go as MSM.

Based on this, all three sites worked on context-specific approaches that would allow them to collaborate with select government health facilities within their respective counties. In Kisumu for instance, apart from their clinic operating within the CBO premises, the organisation negotiated and was assigned space at one of the government facilities in order to offer HIV prevention and treatment services including HIVST kits to their members. 
Alongside the integration of study findings to ongoing interventions, it is important to note the implementation of the intervention was equally an iterative process which involved several revisions being made to the intervention strategies as findings emerged. For instance, as captured in the ethnographic field notes, in Mombasa, because of COVID-19, they engaged a motorbike rider to ensure continuity of distribution of the HIV self-testing kits to the MSM community within the target area. Also, in all the sites, demand creation through the social media and other virtual spaces was intensified.

\section{Discussion}

Implementing the HIVST intervention while following the principles of CBPS promoted meaningful collaborations and a mutually empowering process across the diverse stakeholders. Specifically, the process enabled the community to gain the confidence, authority and capability to meaningfully contribute to the entire research process. Active participation of the community in all phases of the research project, including shaping the direction of the study and the analysis of findings, realizes the central tenants of community-based research 68,69 . Such inclusion promotes co-learning and empowers the community to steer social change, and enables the researchers to implement studies that are grounded in real contexts 70-72. The CBOs implementing the HIVST intervention, by virtue of playing an integral part in the analysis process, were able to translate the findings to allow the integration of HIVST in their ongoing HIV prevention and treatment programs. In this way, $\mathrm{CBO}$ s readily adopted the qualitative evidence into their program activities.

While meaningful community inclusion is a key component of CBPS projects, as is the case with other forms of CBR, there are inherent power imbalances that exist between the 'researchers' and the 'community'. Even with well-designed and well-intentioned studies aiming for equal partnerships and sharing of power in the co-production of evidence, disrupting traditional power hierarchies can still be challenging 73. In our example, the fact that UM was the main applicant to the research funding and interacted with the donors, while the CBOs were not aware of the UM's budget but named as coinvestigators in the study, helped maintain the traditional power hierarchies. As a result, we observed that the CRs frequently waited or asked for direction from the 'researchers' rather than taking the lead in the discharge of their roles. In addition, the community always expressed their gratitude for the opportunities that the 'researchers' provided to them, while negating to acknowledge the opportunities that they themselves provided to the researchers, including the platform in which the research took place. It remains a challenge to create a sense of 'equal' partnership among community and academic researchers in studies, with the unique knowledge and valuable contribution by community often failing to be significantly recognized by both the community themselves and other members of the research team. In our project, efforts were made by the researchers to organize the research training sessions in a way that was participatory to facilitate 'equal' and bi-directional learning for both the community and the researchers.

Having community members as part of the research team has been promoted as one way to help address researcher-researched power imbalances, potentially leading to higher levels of community acceptance 
and the community actively contributing their local, specialized knowledge during study implementation5,74. There has been increasing attention given to building balanced partnerships in research between researchers and marginalized communities 12,15,75. In some instances, researchers and community activists have together launched advocacy projects as a way to ensure that members of the MSM community are actively engaged in research studies. This is done with the primary aim of strengthening the capacity of the community, as well as establishing the legitimacy of the research team as a whole such that research conducted is acceptable by the community $76-80$.

As described earlier, through the MSM-led CBOs, community members were recruited as researchers from within the membership of the $\mathrm{CBOs}$. Although the community researchers were carefully trained on research ethics and performed several role-playing exercises based on potential scenarios in the field; in practice, dealing with challenges related to the dual role of 'researcher' and 'community member' at times caused more discomfort than envisioned. When doing the interviews, the community researchers were 'out' about being part of the MSM community, a move that was thought would foster a level of trust with the participants. However, some community researchers reported receiving sexual propositions from their participants which made the progress of their interviews challenging and might have affected the nature of the responses from the participants. Ibáñez-Carrasco and colleagues recommend research teams prepare peer researchers for ongoing changes that may occur in their roles, identity and experience. 81 In our context, the team discussed such potential occurrences and agreed it was appropriate to discontinue an interview with a participant that made them feel uncomfortable, or to clearly explain to the participants the boundaries between their work and social lives. This helped the CRs to maintain a sense of professionalism needed to safely and comfortably complete their work, while interacting with their peer participants.

\section{Conclusion}

A CBPS approach aims to ensure that communities are not only 'collaborators' but 'colleagues' in the production of evidence and the overall implementation of the study. We emphasize the importance of creating operating norms and clear definition of partnership roles well in advance and before embarking on the study as an important step to take. We observed that such a move in CBPS gives the community a chance to trust their capabilities and more equally contribute to the implementation of the study. Over and above the fact that we observed power imbalances play out in the implementation of our CBPS HIVST study, power hierarchies were effectively challenged through clear role definitions set up during the study design. All partners were part of the early discussions about the study and, as a result, were clear on their roles in the HIVST implementation and integration of study findings into existing community programs. Other researchers taking up CBPS as an approach should consider allowing time for building meaningful relationships with the relevant communities and other stakeholders as a way of beginning to build a platform where all partners feel valuable to contribute their knowledge and expertise.

\section{Abbreviations}


AIDS

Acquired Immune Deficiency Syndrome

CBO

Community-Based Organization

CBPS

Community-based programme science

CBR

Community-based research

CR

Community Researchers

FSW

Female Sex Worker

HAPA Kenya

The HIV \& AIDS People's Alliance of Kenya

HIV

Human Immuno-Deficiency Virus

HIVST

HIV Self-Test

LGBTI

Lesbian, Gay, Bisexual, Transgender, Intersex

MAAYGO

Men Against AIDS Youth Group

MPEG

Mamboleo Peer Empowerment Group

MSM

Men Who Have Sex with Men

NACC

National AIDS Control Council

NASCOP

National AIDS and STI Control Programme

NGO

Non-governmental organisations

PHDA

Partners for Health and Development in Africa

UM

University of Manitoba.

\section{Declarations}


The study obtained approval from the institutional review boards of the Kenyatta National Hospital University of Nairobi, Kenya (P557/08/2018) and the University of Manitoba-Health Research Ethics Board, Canada (HS22205). All participants provided written informed consent.

\section{Adherence to national and international regulations}

Not applicable

\section{Consent for publication}

All participants provided written informed consent before participating in the study, which included consent to publish anonymous quotes from individual participants.

\section{Availability of data and material}

The datasets that support the findings of this study are available from the corresponding author on reasonable request.

\section{Competing interests}

The authors declare that they have no competing interests.

\section{Funding}

This publication is made possible by the support of Bill \& Melinda Gates Foundation (BMGF) under grant OPP-11191068. The views expressed herein are those of the authors and do not necessarily reflect the official policy or position of BMGF.

\section{Authors' contributions}

PB, RL, BK, MT, LL, HM, KO, SK, MK, MO, MN, and JM participated in the design of the study, designed the qualitative and quantitative study and its instruments and drafted the manuscript. BK, RL and MT trained the community researchers in qualitative data collection and analysis. RL, MT, BK, KO, SK, MO, MN analyzed the qualitative data. $\mathrm{PB}, \mathrm{HM}, \mathrm{JM}, \mathrm{MO}, \mathrm{MN}$ designed the intervention. BK drafted the first draft of the manuscript. MT, LL, PB, RL reviewed the manuscript. All authors have read and approved the final manuscript.

\section{Acknowledgements}

The authors would like to thank the local research team which included Juddie Onyoni, Memory Melon, Margret Njraini, Japheth Kioko and Shem Kaosa. We would also like to thank all the participants of the protocol development workshop conducted in July 2018, and the community researchers and research assistants from the three participating sites who supported data collection and even data analysis for the qualitative team. 


\section{References}

1. Keikelame MJ, Swartz L. Decolonising research methodologies: lessons from a qualitative research project, Cape Town, South Africa. Global Health Action. 2019;12(1).

2. Thambinathan V, Kinsella EA. Decolonizing Methodologies in Qualitative Research: Creating Spaces for Transformative Praxis: https://doi.org/101177/16094069211014766. 2021 May 4;20.

3. Held MBE. Decolonizing Research Paradigms in the Context of Settler Colonialism: An Unsettling, Mutual, and Collaborative Effort. International Journal of Qualitative Methods. 2019 Jan 21;18.

4. Drawson AS, Toombs E, Mushquash CJ. Indigenous research methods: A systematic review. International Indigenous Policy Journal. 2017;8(2).

5. Israel BA, Schulz AJ, Coombe CM, Parker EA, Reyes AG, Rowe Z, et al. Community-Based Participatory Research. In: Urban Health. 2019. p. 272-82.

6. Minkler M, Garcia AP, Rubin V, Wallerstein N. Community-Based Participatory Research: A Strategy for Building Healthy Communities and Promoting Health through Policy. 2012.

7. Blanchard AK, Nair SG, Bruce SG, Aids C, Sangha TM, Ramanaik S, et al. A community-based qualitative study on the experience and understandings of intimate partner violence and HIV vulnerability from the perspectives of female sex workers and male intimate partners in North Karnataka state, India. BMC Women's Health. 2018;18:66.

8. Blanchard AK, Nair SG, Thalinja R, Srikantamurthy HS, Ramanaik S, Javalkar P, et al. Pursuing Authenticity from Process to Outcome in a Community-Based Participatory Research Study of Intimate Partner Violence and HIV Vulnerability in North Karnataka, India. Qualitative Health Research. 2017 Jan 1;27(2):204-14.

9. Israel BA, Schulz AJ, Parker EA, Becker AB. Review of community-based research: assessing partnership approaches to improve public health. Annu Rev Public Health. 1998;19:173-202.

10. Bidwell D. Is community-based participatory research postnormal science? Science Technology Human Values. 2009;34(6):741-61.

11. O'Fallon LR, Dearry A. Community-based participatory research as a tool to advance environmental health sciences. Environ Health Perspect. 2002;110(SUPPL. 2):155-9.

12. Ziegler TB, Coombe CM, Rowe ZE, Clark SJ, Gronlund CJ, Lee M, et al. Shifting from "communityplaced" to "community-based" research to advance health equity: A case study of the heatwaves, housing, and health: Increasing climate resiliency in Detroit $(\mathrm{HHH})$ partnership. International Journal of Environmental Research and Public Health. 2019.

13. Balazs CL, Morello-Frosch R. The Three Rs: How Community-Based Participatory and Reach of Science. Environemental Justice. 2013.

14. Kraemer Diaz AE, Spears Johnson CR, Arcury TA. Perceptions That Influence the Maintenance of Scientific Integrity in Community-Based Participatory Research. Health Education and Behavior. 2015. 
15. Brizay U, Golob L, Globerman J, Gogolishvili D, Bird M, Rios-Ellis B, et al. Community-academic partnerships in HIV-related research: A systematic literature review of theory and practice. Journal of the International AIDS Society. 2015.

16. Israel BA, Schulz AJ, Parker EA, Becker AB. REVIEW OF COMMUNITY-BASED. RESEARCH: Assessing Partnership Approaches to Improve Public Health. Annu Rev Public Health. 1998;19(1):173-202.

17. Aral SO, Blanchard JF. The program science initiative: Improving the planning, implementation and evaluation of HIV/STI prevention programs. Sexually Transmitted Infections. 2012 Apr;88(3):157-9.

18. Blanchard JF, Aral SO. Program science: An initiative to improve the planning, implementation and evaluation of HIV/sexually transmitted infection prevention programmes. Sexually Transmitted Infections. 2011;Vol. 87:2-3.

19. Becker M, Mishra S, Aral S, Bhattacharjee P, Lorway R, Green K, et al. The contributions and future direction of Program Science in HIV/STI prevention. Emerging Themes in Epidemiology. 2018 May 28;15(1):7.

20. Crockett M, Avery L, Blanchard J. Program science-A framework for improving global maternal, newborn, and child health. Vol 169, JAMA Pediatrics. American Medical Association; 2015. pp. 3056.

21. Kothari A, Rudman D, Dobbins M, Rouse M, Sibbald S, Edwards N. The use of tacit and explicit knowledge in public health: A qualitative study. Implementation Science. 2012 Mar;20(1):20. 7 (.

22. Baral S, Sifakis F, Cleghorn F, Beyrer C. Elevated risk for HIV infection among men who have sex with men in low- and middle-income countries 2000-2006: A systematic review. PLoS Medicine. 2007.

23. Sanders EJ, Graham SM, Okuku HS, Van Der Elst EM, Muhaari A, Davies A, et al. HIV-1 infection in high risk men who have sex with men in Mombasa, Kenya. AIDS. 2007 Dec;21(18):2513-20.

24. Sanders EJ, Okuku HS, Smith AD, Mwangome M, Wahome E, Fegan G, et al. High HIV-1 incidence, correlates of HIV-1 acquisition, and high viral loads following seroconversion among MSM. AIDS. 2013 Jan;28(3):437-46. 27(.

25. NASCOP. Key Population Mapping and Size Estimation in Selected Counties in Kenya: Phase 1 KEY FINDINGS NATIONAL AIDS AND STI CONTROL PROGRAMME (NASCOP). 2019.

26. NASCOP. Kenya. HIV Prevention Response and Modes of Tranmission Study. Ministry of Health. 2013.

27. National Key Populations Programme NM of HK. 2010-2011 Integrated biological and behavioural surveillance survey among key populations in Nairobi and Kisumu, Kenya. 2014.

28. Fay H, Baral SD, Trapence G, Motimedi F, Umar E, lipinge S, et al. Stigma, health care access, and HIV knowledge among men who have sex with men in Malawi, Namibia, and Botswana. AIDS Behav. 2011 Aug;15(6):1088-97.

29. Okall DO, Ondenge K, Nyambura M, Otieno FO, Hardnett F, Turner K, et al. Men Who Have Sex With Men in Kisumu, Kenya: Comfort in Accessing Health Services and Willingness to Participate in HIV Prevention Studies. J Homosex. 2014 Dec;61(12)(1):1712-26. 
30. (No Title).

31. UNAIDS. ACT TO CHANGE LAWS THAT DISCRIMINATE. 2019.

32. Legal G. Criminal Laws on Homosexuality in African Nations. Vol 6462, Global Research Directorate Staff. The Law Library of Congress; 2014.

33. Homosexuality remains illegal in Kenya as. court rejects LGBT petition [Internet]. [cited 2020 Jul 31]. Available from: https://theconversation.com/homosexuality-remains-illegal-in-kenya-as-court-rejectslgbt-petition-112149.

34. Awondo P, Geschiere P, Reid G. Homophobic Africa? Toward A More Nuanced View. Afr Stud Rev. 2012;55(3):145-68.

35. Golub SA, Gamarel KE. The impact of anticipated HIV stigma on delays in HIV testing behaviors: Findings from a community-based sample of men who have sex with men and transgender women in New York City. AIDS Patient Care and STDs. 2013 Nov 1;27(11):621-7.

36. Wei C, Cheung DH, Yan H, Li J, Shi LE, Raymond HF. The Impact of Homophobia and HIV Stigma on HIV Testing Uptake among Chinese Men Who Have Sex with Men: A Mediation Analysis. Jan 1: Journal of Acquired Immune Deficiency Syndromes Lippincott Williams and Wilkins; 2016. pp. 8793.

37. Fauk NK, Merry MS, Sigilipoe MA, Putra S, Mwanri L. Culture, social networks and HIV vulnerability among men who have sex with men in Indonesia. Garcia J, editor. PLOS ONE. 2017 Jun 5;12(6):e0178736.

38. Gamarel KE, Nelson KM, Stephenson R, Santiago Rivera OJ, Chiaramonte D, Miller RL. Anticipated HIV Stigma and Delays in Regular HIV Testing Behaviors Among Sexually-Active Young Gay, Bisexual, and Other Men Who Have Sex with Men and Transgender Women. AIDS and Behavior. 2018 Feb 1;22(2):522-30.

39. Logie CH, Newman PA, Weaver J, Roungkraphon S, Tepjan S. HIV-related stigma and HIV prevention uptake among young men who have sex with men and transgender women in Thailand. AIDS Patient Care and STDs. 2016 Feb 1;30(2):92-100.

40. S M, M B, C M, W V, R P. A review of HIV testing strategies among MSM (2005-2015): Changes and continuities due to the biomedicalization of responses to AIDS. Global public health. 2019 May 4;14(5):764-76.

41. Mora C, Brigeiro M, Monteiro SHIV, Testing Among. "MSM": Prevention Technologies, Sexual Moralities and Serologic Self-surveillance. Physis: Revista de Saúde Coletiva. 2018 Aug $13 ; 28(2): 280204$.

42. Council P. FEASIBILITY AND ACCEPTABILITY OF HIV SELF-TESTING AMONG. MEN WHO HAVE SEX WITH MEN IN NIGERIA. 2018.

43. Okoboi S, Lazarus O, Castelnuovo B, Nanfuka M, Kambugu A, Mujugira A, et al Peer distribution of HIV self-test kits to men who have sex with men to identify undiagnosed HIV infection in Uganda: A pilot study. Clark JL, editor. PLOS ONE. 2020 Jan 23;15(1):e0227741. 
44. Figueroa C, Johnson C, Verster A, Baggaley R. Attitudes and Acceptability on HIV Self-testing Among Key Populations: A Literature Review.

45. Witzel TC, Rodger AJ, Burns FM, Rhodes T, Weatherburn P. HIV Self-Testing among Men Who Have Sex with Men (MSM) in the UK: A Qualitative Study of Barriers and Facilitators, Intervention Preferences and Perceived Impacts. Paraskevis D, editor. PLOS ONE. 2016 Sep 9;11(9):e0162713.

46. Johnson CC, Kennedy C, Fonner V, Siegfried N, Figueroa C, Dalal S, et al. Examining the effects of HIV self-Testing compared to standard HIV testing services: A systematic review and meta-Analysis. Journal of the International AIDS Society [Internet]. 2017 [cited 2020 Jul 31];20(1). Available from: https://pubmed.ncbi.nlm.nih.gov/28530049/.

47. Chipungu J, Bosomprah S, Zanolini A, Thimurthy H, Chilengi R, Sharma A, et al. Understanding linkage to care with HIV self-test approach in Lusaka, Zambia - A mixed method approach. PLoS ONE. 2017 Nov 1;12(11).

48. Njau B, Covin C, Lisasi E, Damian D, Mushi D, Boulle A, et al. A systematic review of qualitative evidence on factors enabling and deterring uptake of HIV self-testing in Africa. BMC Public Health. 2019 Oct 15;19(1):1289.

49. Masters SH, Agot K, Obonyo B, Napierala Mavedzenge S, Maman S, Thirumurthy H. Promoting Partner Testing and Couples Testing through Secondary Distribution of HIV Self-Tests: A Randomized Clinical Trial. PLoS Medicine. 2016 Nov 1;13(11).

50. Agot K, et al. Perceptions of adolescent girls about their ability to offer HIV self-test kits to their sexual partners: a pilot study in Siaya County, western Kenya | HIVST.org [Internet]. 2017 [cited 2020 Jul 31]. Available from: http://hivst.org/evidence/perceptions-of-adolescent-girls-about-their-abilityto-offer-hiv-self-test-kits-to-their-sexual-partners-a-pilot-study-in-siaya-county-western-kenya.

51. Kelvin EA, George G, Mwai E, Kinyanjui S, Romo ML, Odhiambo JO, et al. A Randomized Controlled Trial to Increase HIV Testing Demand Among Female Sex Workers in Kenya Through Announcing the Availability of HIV Self-testing Via Text Message. AIDS and Behavior. 2019 Jan 15;23(1):116-25.

52. Agot K, et al. Secondary distribution of HIV self-tests as a way to promote HIV testing among male partners of young women: subgroup analysis from a randomized trial | HIVST.org [Internet]. 2018 [cited $2020 \mathrm{Jul}$ 31]. Available from: http://hivst.org/evidence/secondary-distribution-of-hiv-self-testsas-a-way-to-promote-hiv-testing-among-male-partners-of-young-women-subgroup-analysis-from-arandomized-trial.

53. Athowe AE. Uptake of HIV oral self-testing among female sex workers in Starehe Nairobi: lessons from the key population implementation science study I HIVST.org [Internet]. 2018 [cited 2020 Jul 31]. Available from: http://hivst.org/evidence/uptake-of-hiv-oral-self-testing-among-female-sexworkers-in-starehe-nairobi-lessons-from-the-key-population-implementation-science-study.

54. Achia, et al. The role of oral-HIV self-testing in enrolment of female sex workers into comprehensive HIV programmes in Kenya | HIVST.org [Internet]. 2018 [cited 2020 Jul 31]. Available from: http://hivst.org/evidence/the-role-of-oral-hiv-self-testing-in-enrolment-of-female-sex-workers-intocomprehensive-hiv-programmes-in-kenya. 
55. van der Elst EM, Clifford MS, O C O, F I BK. Peer-led oral HIV-self testing finds undiagnosed HIV among MSM in Malindi, Kenya I HIVST.org [Internet]. 2017 [cited 2020 Jul 31]. Available from: http://hivst.org/evidence/peer-led-oral-hiv-self-testing-finds-undiagnosed-hiv-among-msm-in-malindikenya.

56. Bhattacharjee P, Rego D, Musyoki H, Becker M, Pickles M, Isac S, et al. Evaluation of communitybased HIV self-testing delivery strategies on reducing undiagnosed HIV infection, and improving linkage to prevention and treatment services, among men who have sex with men in Kenya: $A$ programme science study protocol. BMC Public Health. 2019 Jul 23;19(1):986.

57. Emmanuel F, Kioko J, Musyoki H, Kaosa S, Ongaro MK, Kuria S, et al. Mapping virtual platforms to estimate the population size of men who have sex with men (MSM) who use internet to find sexual partners: implications to enhance HIV prevention among MSM in Kenya. Gates Open Research. 2020 Dec;10:4:131.

58. Anderson EE. Cirtification: Training in human research protections for community-engaged research partners. Progress in Community Health Partnerships: Research, Education, and Action. 2015 Dec $1 ; 9(2): 283-8$.

59. B JJ,PL, M JS,AC, G T. G. W, et al. A realist evaluation of community-based participatory research: partnership synergy, trust building and related ripple effects. BMC public health. 2015.

60. Flicker S. Who benefits from community-based participatory research? A case study of the positive youth project. Health Education and Behavior. 2008.

61. Springer MV, Skolarus LE. Community-based participatory research: Partnering with communities. Stroke. 2019.

62. Ambrosini V, Bowman C. Tacit Knowledge: Some Suggestions for Operationalization. Journal of Management Studies. 2001 Sep 1;38(6):811-29.

63. Lorway R, Doshi M, Mathenge J, Macharia P, McKinnon L, Bhattacharjee P, et al. Constructing "problem drinking" and HIV vulnerability with male sex workers in Nairobi: a participatory study. In: Sandfort T, Aggleton P, editors. Men who have sex with men and HIV vulnerability in Africa. New York: Routledge; 2020.

64. Lorway R, Thompson LH, Lazarus L, du Plessis E, Pasha A, Fathima Mary P, et al. Going beyond the clinic: confronting stigma and discrimination among men who have sex with men in Mysore through community-based participatory research. Critical Public Health. 2014 Jan;24(1):73-87.

65. Ryan G. Introduction to positivism, interpretivism and critical theory. Nurse Researcher. 2018.

66. Odinga MM, Kuria S, Muindi O, Mwakazi P, Njraini M, Melon M, et al. HIV testing amid COVID-19: community efforts to reach men who have sex with men in three Kenyan counties. Gates Open Research [Internet]. 2020 Oct 29 [cited 2021 Aug 19];4:117. Available from: /pmc/articles/PMC7477340/.

67. Minkler M, Wallerstein N. Community-Based Participatory Research for Health: from Process to Outcomes. 2nd ed. San Franscisco: Jossey-Bass; 2008. 
68. Israel BA, Schulz AJ, Parker EAA, Becker AB. Review of community-based research: assessing partnership approaches to improve public health. Health Behavior and Health Education, University of Michigan School of Public Health, 1420 Washington Heights. Annu Rev Public Health. 1998;19:173202.

69. Fawcett SB, Paine-Andrews A, Francisco VT, Schultz JA, Richter KP, Lewis RK, et al. Using empowerment theory in collaborative partnerships for community health and development. Am J Community Psychol. 1995 Oct;23(5):677-97.

70. Laverack G. Improving health outcomes through community empowerment: A review of the literature. Journal of Health Population Nutrition. 2006;Vol. 24:113-20.

71. Hughey J, Peterson NA, Lowe JB, Oprescu F. Empowerment and sense of community: Clarifying their relationship in community organizations. Health Education and Behavior. 2008 Oct;35(5):651-63.

72. Gibson A, Britten N, Lynch J. Theoretical directions for an emancipatory concept of patient and public involvement. Health. 2012 Sep;16(5):531-47.

73. Israel BA, Coombe CM, Cheezum RR, Schulz AJ, McGranaghan RJ, Lichtenstein R, et al. Communitybased participatory research: A capacity-building approach for policy advocacy aimed at eliminating health disparities. Am J Public Health. 2010;100(11):2094-102.

74. Funnell S, Tanuseputro P, Letendre A, Bearskin LB, Walker J. "Nothing About Us, without Us." How Community-Based Participatory Research Methods Were Adapted in an Indigenous End-of-Life Study Using Previously Collected Data. Canadian journal on aging = La revue canadienne du vieillissement. 2020.

75. Harris DA, Pensa MA, Redlich CA, Pisani MA, Rosenthal MS. Community-based participatory research is needed to address pulmonary health disparities. Vol 13, Annals of the American Thoracic Society. American Thoracic Society; 2016. pp. 1231-8.

76. Skolarus LE, Zimmerman MA, Murphy J, Brown DL, Kerber KA, Bailey S, et al. Community-based participatory research: A new approach to engaging community members to rapidly call 911 for stroke. Stroke. 2011.

77. Munoz R, Fox MD. Research impacting social contexts: The Moral Import of Community-Based participatory research. Vol. 11: American Journal of Bioethics. Taylor \& Francis Group; 2011. pp. 378.

78. Tobias JK, Richmond CAM, Luginaah I. Community-based participatory research (CBPR) with indigenous communities: Producing respectful and reciprocal research. Journal of Empirical Research on Human Research Ethics. 2013.

79. Ramsden VR, Mckay S, Crowe J. The pursuit of excellence: Engaging the community in participatory health research. Global Health Promotion. 2010.

80. Ibáñez-Carrasco F, Watson JR, Tavares J. Supporting peer researchers: Recommendations from our lived experience/expertise in community-based research in Canada. Vol. 16: Harm Reduction Journal. BioMed Central Ltd.; 2019. p. 55. 
Figures

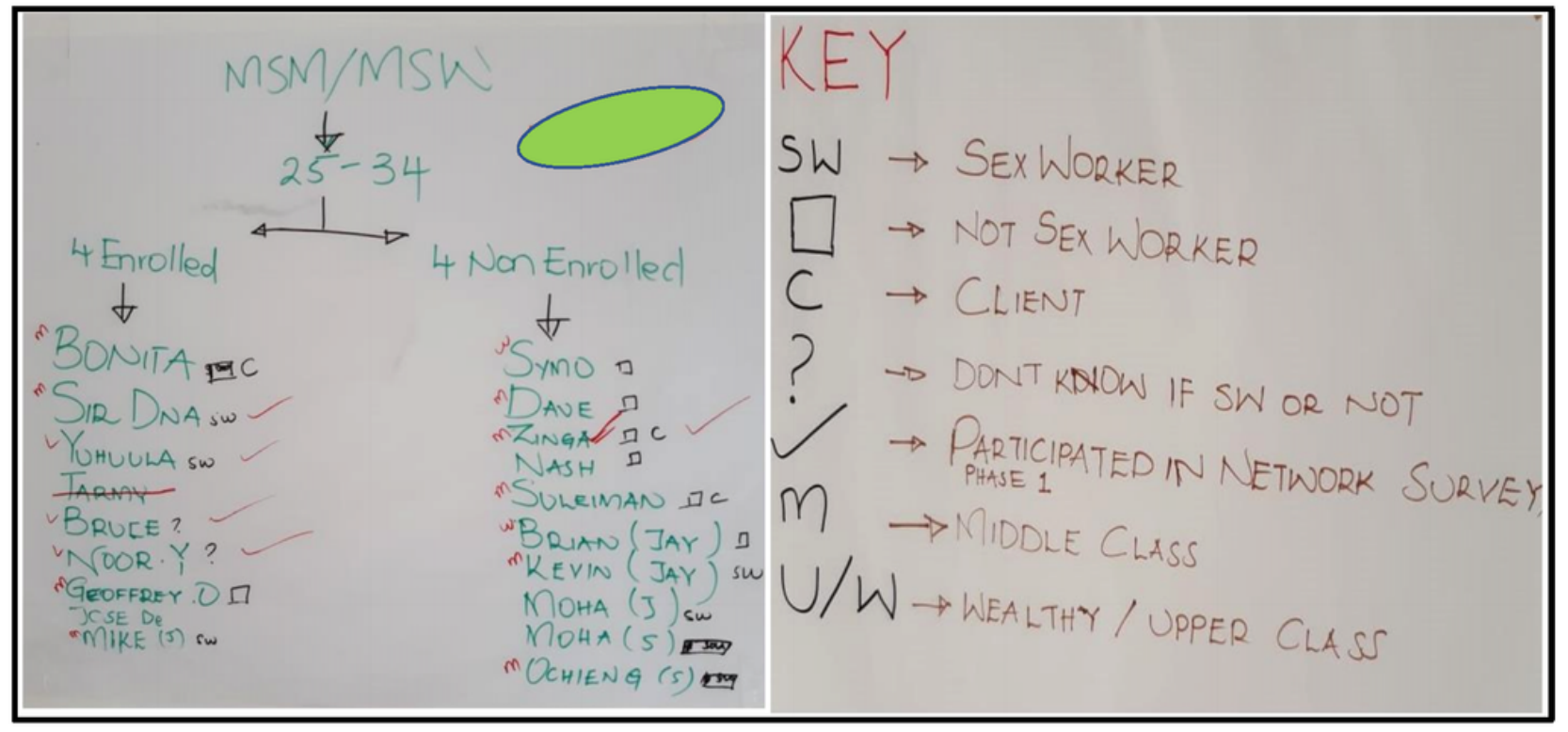

Figure 1

Sampling tree example with pseudonyms developed by community researchers at respective sites 

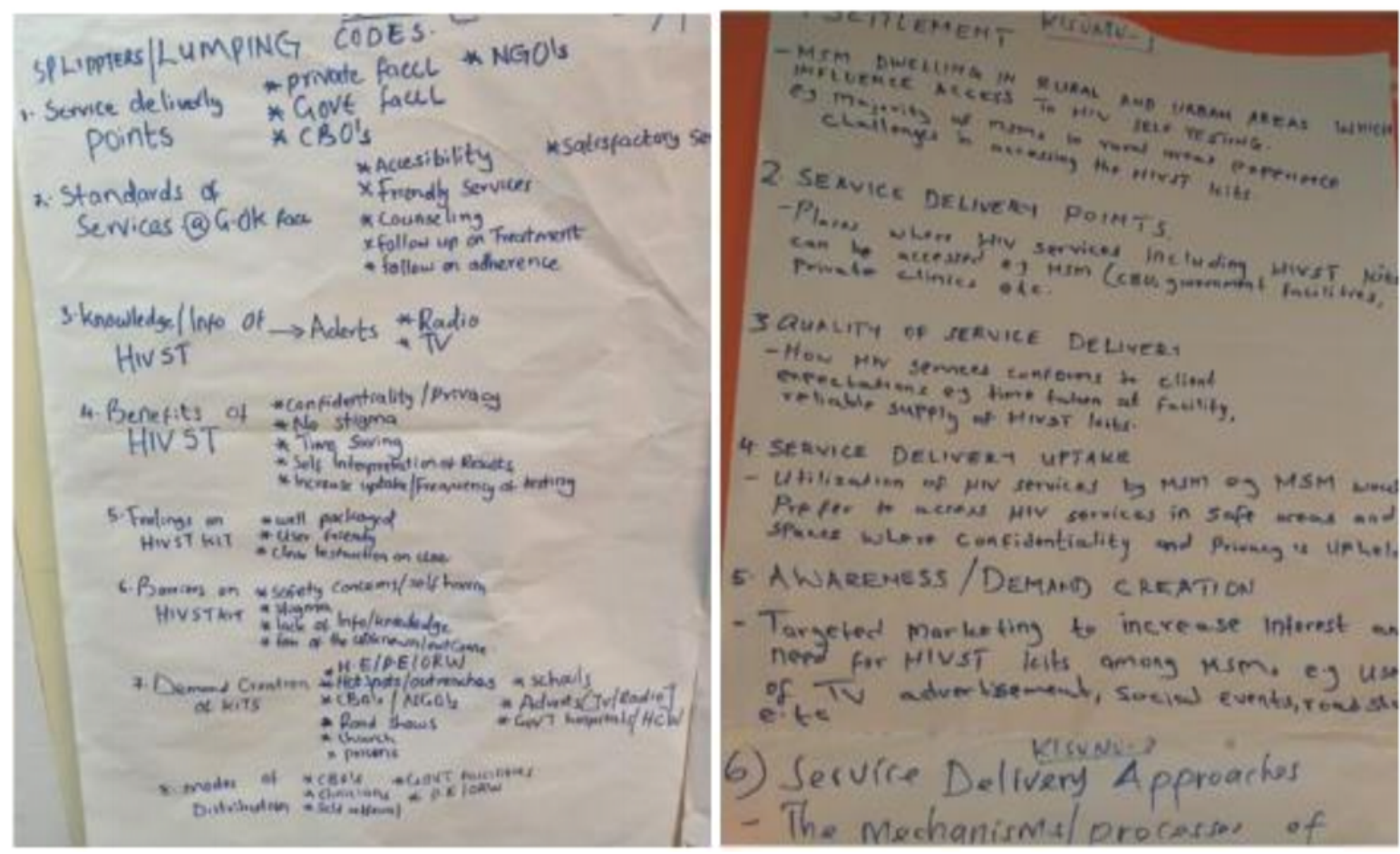

Figure 2

Sample of child codes to make parents codes and consolidated code definitions 